\title{
Molecular Signaling Networks That Choreograph Epimorphic Fin Regeneration in Zebrafish - A Mini-Review
}

\author{
Tamara L. Tal Jill A. Franzosa Robert L. Tanguay \\ Department of Environmental and Molecular Toxicology, Environmental Health Sciences Center, \\ Oregon State University, Corvallis, Oreg., USA
}

\section{Key Words}

Regeneration - Zebrafish - Wnt • Fibroblast growth factor • Activin- $\beta A \cdot$ Retinoic acid $\cdot$ Sonic hedgehog $\cdot$ MicroRNA

\begin{abstract}
This short review provides a current synopsis of caudal fin regeneration in zebrafish with an emphasis on the molecular signaling networks that dictate epimorphic regeneration. At the outset, the fundamentals of caudal fin architecture and the stages of epimorphic regeneration are described. This is followed by a detailed look at the main networks implicated in fin regeneration, namely the Wnt, fibroblast growth factor, activin- $\beta A$, retinoic acid and hedgehog signaling pathways. Throughout this mini-review, these molecular networks are examined through the lens of wound healing, blastema formation or regenerative outgrowth, three of the main stages of epimorphic regeneration. Next, the emerging role of noncoding RNAs as regulators of regeneration and mechanisms of regenerative termination are discussed. Finally, the implications for future research and the broader field of regenerative medicine are examined.
\end{abstract}

Copyright $\odot 2009$ S. Karger AG, Basel

\section{Introduction}

Regeneration is a research area that has captivated the imagination of biologists for decades. Mammals can regenerate a limited number of structures including skin, digits, skeletal muscle, hair cells, gut epithelium, liver, pancreas and blood. However, mammalian central nervous system tissues and structures (brain, retina and spinal cord) and heart, limb and kidney all fail to regenerate. Therefore, numerous human conditions resulting from injury, aging and disease could be significantly improved if therapies that encourage tissue regeneration were available. The field of regenerative medicine is aimed at developing strategies to restore individual cell types, complex tissues or structures that are lost or damaged. This emerging field is approached from two distinct angles. In recent years, stem cell-based models have been developed to generate a suite of differentiated cells for therapeutic applications. The alternative approach employs the inherent regenerative capacity of nonmammalian models to define the molecular events that permit tissue regeneration. While there are several regenerative animal models including salamanders, newts, hydra and flatworms that are established to evaluate tissue regeneration, zebrafish have emerged as a powerful vertebrate regenerative model because of advances in zebrafish genetics, rapid regeneration time, the ability to obtain vast quantities of externally fertilized eggs, and the ability to transiently modify gene function during development. Zebrafish have the remarkable capability to regenerate their heart muscle $[1$, 2], fin [3], retina [4], optic nerve [5], liver [6], spinal cord [7] and sensory hair cells [8]. By understanding the molecular and genetic pathways that coordinately function to accomplish regeneration in this model, we will be in a stronger position to understand why mammals fail to re-

\section{KARGER \\ Fax +4161306 1234 E-Mail karger@karger.ch} www.karger.com (c) 2009 S. Karger AG, Basel

0304-324X/10/0562-0231\$26.00/0

Accessible online at:

www.karger.com/ger
Robert L. Tanguay

Oregon State University

Department of Environmental and Molecular Toxicology, 1007 ALS

Corvallis, OR 97331-7301 (USA)

Tel. +1 541737 6514, Fax +1 541737 7966, E-Mail robert.tanguay@ oregonstate.edu 
spond to tissue injury with a regenerative response. This will lead to the generation of therapeutic interventions designed to circumvent limitations in mammalian regenerative abilities.

\section{Conserved Signaling Pathways in Regenerating Tissues}

Well-defined signaling molecules are similarly involved in regulating appendage development in a number of species from flies to humans [9]. While there are obvious differences in size, shape and function among these nonhomologous appendages, what is more interesting from a developmental perspective is what signaling mechanisms are conserved among them. For example, key members of the fibroblast growth factor (FGF) and wingless (Wnt) families are well-defined players in human, mouse, chicken, tadpole and fruit fly limb development [10-12], suggesting that while final limb structures might vary in form and function, the molecular networks used to create these appendages are conserved across multiple species.

In the case of limbs or fins, the signaling pathways that initiate and maintain appendage development are reactivated to promote regeneration in certain nonmammalian species following amputation. In addition, conservation of morphological and molecular events among different regenerating systems within the same species has been extensively reported. For example, there is growing evidence to suggest that zebrafish heart and fin regeneration have a high degree of commonality with respect to key signaling pathways that are involved in promoting regeneration following a surgical wound. The nightcap zebrafish mutant, harboring a temperature-sensitive mutation in the mpsl gene, and the nbl (no blastema) zebrafish mutant, containing a missense mutation in hsp60, fail to regenerate both heart and fin tissue [1, 13, 14]. Mps1 and hsp60 are also required for regeneration of light-damaged retinal neurons [15]. In addition, knockdown of FGF signaling using a dominant negative FGF receptor 1 (fgfrl) transgenic line impaired both heart and caudal fin regeneration through impairment of revascularization or blastemal proliferation, respectively $[16,17]$. At the gene expression level, Lien et al. [18] showed that 132 out of 662 genes that are differentially expressed in regenerating heart tissue are similarly up- or downregulated in regenerating fins. However, the same study noted that 491 and 892 genes were differentially expressed in either regenerating heart or fin tissue, respectively $[18,19]$. In situ expression analysis has also been used to demonstrate conservation of spatial and temporal gene expression in different regenerating tissues. For example, expression patterns of notchlb and deltaC, members of the Notch signaling pathway, are induced early after heart amputation, as well as in the regenerating fin blastema, but not in nonamputated fin or heart tissue [20]. Expression of simplet (smp), a gene associated with the regulation of cell proliferation, has been similarly localized to regenerating heart and fin tissue [21]. Taken together, these studies support the concept that a common group of signaling molecules are likely involved in both fin and heart regeneration but that many tissue-specific factors are also required. This is not surprising, considering that cardiac regeneration stems from cardiomyocyte proliferation [16] while fin regeneration is achieved via dedifferentiation of mesenchymal cells to form a mass of undifferentiated proliferative cells called a blastema [reviewed in 13]. Although zebrafish can regenerate a diverse array of complex tissue types, the caudal fin regeneration model is primarily used to investigate regenerative mechanisms because of the ability to perform easy external surgeries, symmetric fin architecture, limited cell type composition and rapid regeneration time.

\section{Caudal Fin Architecture}

Zebrafish caudal fins are complex structures that contain 16-18 lepidotrichia (fin rays) connected by soft tissue interrays that lack skeletal elements. The fin rays are a series of bony segments comprised of a pair of concave hemirays surrounded by a monolayer of scleroblasts (bone-secreting cells). The hemirays function to protect an intraray core consisting of mesenchymal cells, blood vessels, nerves, melanocytes and fibroblasts. Mesenchymal compartmental components are also present in the interray space. The entire multi-ray fin is covered by an epithelial cell layer. The fin displays an indeterminate ontogenetic growth pattern, meaning that fin growth occurs by the gradual addition of bony ray segments to the distal tip of the fin.

\section{Stages of Epimorphic Fin Regeneration}

Upon caudal fin amputation, a remarkable series of regenerative stages are initiated that result in the complete restoration of lost bone, epidermis, blood vessels, nerves, connective tissue and pigmentation (fig. 1). This complex process, termed epimorphic regeneration, is 
completed in approximately 3 days in larval fish and 2 weeks in adult fish [reviewed in 22]. Epimorphic regeneration is a term used to describe a regenerative process involving the formation of a mass of undifferentiated proliferative multipotent mesenchymal cells called a blastema [23]. In the adult model, an initial wound healing stage, characterized by nonproliferative lateral epithelial cell migration over the wound and subsequent formation of the apical epidermal cap (AEC), is initiated immediately following surgical removal of caudal fin tissue [24]. Second, the wound epithelium thickens and mesenchymal tissue proximal to the amputation plane begins to disorganize. Cellular disorganization is thought to occur as a result of growth factors that originate from the mature wound epidermis and stimulate mesenchymal cells to dedifferentiate and proliferate as they migrate distally towards the area directly proximal to the AEC [24]. In the third stage, a series of blastemas form at the severed portion of each amputated fin ray. Blastema formation is the main event that distinguishes regeneration from limb development. The blastema is an accumulated mass of progenitor cells that are thought to be pluripotent or able to produce daughter cells capable of differentiating into a variety of cell types required to populate the regenerating tissue. Although pluripotency is inferred from aforementioned migratory and proliferative qualities, lineage-trac-

Fig. 1. Major signaling events that choreograph epimorphic fin regeneration in zebrafish caudal fins. A series of cartoons depicting the longitudinal section of a single adult fin ray during the major stages of regeneration are shown. Arrows do not necessarily imply direct events. a Mature fin ray covered by epithelium containing a series of bony segments that protect a mesenchymal core. b Following amputation, epithelial cells (blue) migrate laterally over the wound to form the AEC. Both Wnt $/ \beta$-catenin and FGF signaling are involved in cell specification during wound healing. c Following formation of the AEC and immature blastema (not pictured), blastemal cells segregate into the distal blastema (purple) and highly proliferative proximal blastema (red). $W n t / \beta$-catenin and FGF signaling are required for blastema formation. miR-203 and miR-133 negatively regulate $\mathrm{Wnt} / \beta$-catenin and FGF signaling pathways, respectively. Overrexpression of wnt5b inhibits blastema formation. d Wnt/ $\beta$-catenin and FGF signaling are required for regenerative outgrowth. wnt $5 \mathrm{~b}$ acts as a negative regulator of canonical Wnt signaling, and wnt5b expression is controlled in part by FGF signaling in the distal epithelium. Activin- $\beta A$ influences patterning and RA, hedgehog, and BMP signaling regulate patterning and bone deposition during regenerative outgrowth. Both Wnt and FGF signaling activate shh, while smp acts as a negative regulator of sh. e FGF signaling influences positional memory and regenerative termination. (Colors in online version only.)

Epimorphic Fin Regeneration in Zebrafish

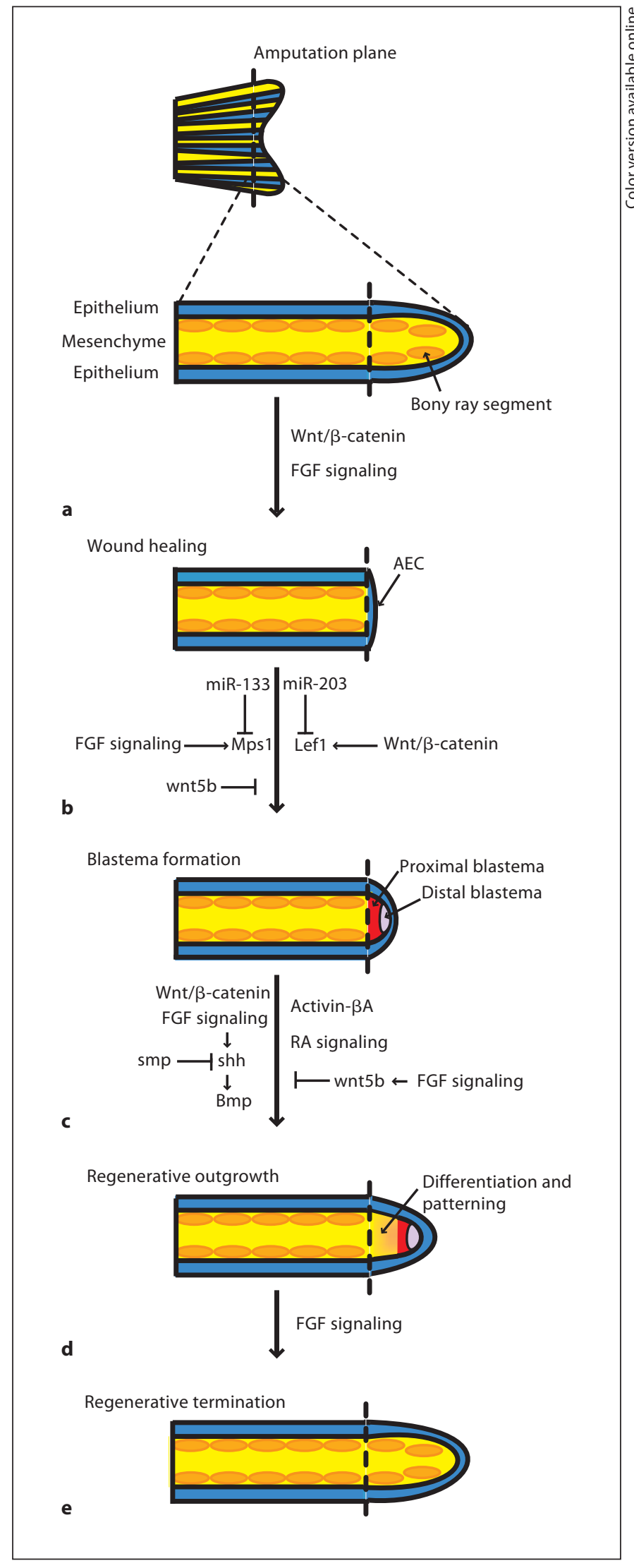

Gerontology 2010;56:231-240 
ing studies have not yet been completed. Therefore, it is possible that resident stem cells or some combination of mesenchymal dedifferentiation and stem cell proliferation give rise to new cells in the regenerating tissue.

The immature blastema proliferates slowly with a median G2 cell cycle time of $>6 \mathrm{~h}$ [24]. Twenty-four hours following caudal fin amputation, blastemal cells segregate into two morphologically identical, but functionally distinct, subpopulations. The distal blastema, located proximally to the AEC, contains msxb-positive cells, proliferates extremely slowly and is hypothesized to specify the regenerating boundary and direct regenerativegrowth [24]. In sharp contrast, the proximal blastema contains a rapidly proliferative mass of msxb-negative cells with a mean G2 time of $<60 \mathrm{~min}$. Together, the proximal and distal blastema form a proliferation gradient with a 50 fold difference in proliferation across an approximate distance of $50 \mu \mathrm{M}$ or 10 cell diameters [24].

The next phase of regenerative outgrowth is marked by intense proliferation in the proximal blastema. A moderately proliferative patterning zone is located immediately proximal to the proximal blastema. The patterning zone contains newly divided cells that migrate to appropriate locations and differentiate to populate the new tissue with mesenchymal cells and fibroblasts. The location and functional differences between the distal and proximal blastema and patterning zone are thought to be generated and maintained through epithelial-mesenchymal interactions $[13,25]$. The final stage, regenerative termination, is not well understood. Fin regeneration proceeds rapidly until the preamputation fin length is reached, at which point it switches to an ontogenetic growth mechanism. It is speculated that termination occurs by either an unknown active termination mechanism or by cessation of regenerative signaling [22]. In an adult fin model, outgrowth occurs from 2 days after amputation (dpa) until the regenerative event is complete at about $14 \mathrm{dpa}$ (at $28^{\circ} \mathrm{C}$ ). Regeneration occurs more quickly at higher temperatures.

\section{Signaling and Epimorphic Regeneration}

The central goal of regeneration research is to elucidate the molecular signaling networks that coordinately function to promote regeneration. In other words, to identify the signaling molecules that induce epithelial wound healing, blastema formation, regenerative outgrowth and termination of regenerative signaling. There has been significant progress in identifying genes and signaling pathways that function during early fin regeneration through the use of mutagenic screens, loss of function and gain of function experiments, gene expression studies and chemical genetics approaches. These approaches have collectively revealed information regarding key pathways that choreograph distinct regenerative stages. The following sections will discuss the main signaling pathways currently known to promote and regulate regenerative signaling in the zebrafish caudal fin (summarized in fig. 1).

\section{Canonical Wnt Signaling Is Required for Wound Healing, Blastema Formation and Regenerative Outgrowth}

Wnts are cysteine-rich secreted glycoprotein ligands that regulate cell fate, polarity, motility and proliferation during development through the activation of multiple intracellular signaling cascades [26]. Wnts bind to members of the Frizzled (FZD) serpentine receptor family to activate canonical signaling that involves $\beta$-catenin or noncanonical signaling that occurs independently of $\beta$ catenin [27]. In the absence of Wnt signaling, cytosolic $\beta$-catenin is complexed with the scaffolding proteins APC, Axin 1, Axin 2 and targeted for rapid proteosomal degradation following phosphorylation by the serine threonine kinases CKI $\alpha$ and GSK3 $\beta[26,28]$. Binding of a Wnt ligand to FZD and low-density-lipoprotein receptor-related protein (LRP) 5/6 coreceptors [29] results in inactivation of the APC-Axin-GSK3 $\beta$ inhibitory complex and stabilization of $\beta$-catenin. Liberated $\beta$-catenin translocates to the nucleus, displaces groucho and HDAC corepressors from the T cell-specific factor/lymphoid enhancer-binding factor 1 (TCF/LEF1) and recruits histone acetylase $\mathrm{CBP} / \mathrm{p} 300$ to activate TCF/LEF1-targeted gene expression [29]. Further regulation of canonical Wnt signaling is conferred by the FZD antagonists Wnt inhibitory factor 1 and secreted FZD-related proteins, both of which compete with Wnts for access to FZDs [30]. Cell surface levels of LRP5/6 are regulated by Dickkopf proteins (Dkks) [31]. Dkks act as LRP antagonists to impair canonical Wnt signaling by reducing LRP5/6 receptor density through the induction of LRP5/6 internalization $[32,33]$. The activity of Dkk is countered by the R-Spondin 1 family of secreted proteins which antagonize Dkkmediated internalization of LRP6 [34].

Wnt signaling was first implicated in zebrafish fin regeneration through the involvement of the Wnt pathway target Lef1 [35]. Lef1 expression was observed in the 
wound epithelium immediately distal to the amputation plane at $12 \mathrm{~h}$ post amputation, suggesting a role for Wnt signaling in subsequent blastema formation. Importantly, treatment with retinoic acid (RA) or the FGF inhibitor SU5402 failed to prevent lef1 expression in the wound epithelium, suggesting that Wnt signaling acts upstream of FGF and RA signaling pathways. In support of these findings, treatment with Dkk1, an inhibitor of canonical Wnt signaling, blocks both fin regeneration and expression of the FGF target Mkp3 [36]. Similarly, overexpression of Dkk1 using a heat shock-inducible transgenic line has been reported to completely inhibit fin regeneration and expression of fgf20 [37]. Interestingly, a regeneration mutant that was identified in a small-scale mutagenesis screen exhibited decreased lef1, mkp3 and msxb expression following amputation [36]. Both the nonregenerative phenoptype and lef1, mkp3 and msxb expression deficiencies were rescued by $\beta$-catenin overexpression. Taken together, these data indicate the importance of canonical Wnt signaling in epimorphic fin regeneration.

The notion that Wnt signaling is required for blastema formation is supported by an elegant series of experiments utilizing adult heat shock-inducible transgenic fish that express Dkk1, a potent inhibitor of canonical Wnt signaling [37]. Inducible expression of Dkk1 was stimulated during each phase of regeneration (0-1 dpa, wound healing; 1-2 dpa, blastema formation; 2-7 dpa, blastema proliferation and regenerative outgrowth) followed by assessment of gross morphology and histology, proliferation and expression of regenerative markers. Heat shock at 0 or $1 \mathrm{dpa}$ prevented formation of the regeneration blastema and expression of lef 1 and $m s x b$, indicating that impairment in Wnt signaling results in the incorrect specification of the blastema mesenchyme and basal epithelial layer [37]. Expression of Dkk1 following blastema formation resulted in marked reduction in epithelial and mesenchymal proliferation, which indicates that Wnt signaling controls blastema maintenance during regenerative outgrowth. Although Wnt/ $\beta$-catenin loss of function studies resulting from overexpression of Dkk fail to prevent lateral epithelial migration over the wound, impairment of Wnt signaling reportedly results in improper specification of the wound epithelium $[36,37]$.

Chemical genetics approaches have further supported the role of Wnt signaling in epimorphic regeneration, demonstrating that both inhibition and inappropriate activation of Wnt signaling similarly result in impaired regenerative abilities [38-40]. Through the use of a diverse synthetic chemical library, a new class of Wnt pathway inhibitors was revealed to negatively impact fin regenera- tion [38]. This class of compounds prevents destruction of Axin proteins, thereby inhibiting liberation of $\beta$ catenin from the APC-Axin-GSK3 $\beta$ inhibitory complex and increasing phosphorylation and subsequent degradation of $\beta$-catenin. Our group has shown that inappropriate activation of Aryl hydrocarbon receptor signaling following 2, 3, 7, 8-tetrachlorodibenzo-p-dioxin (TCDD) exposure impairs tissue regeneration in larval and adult zebrafish $[41,42]$. Comparative toxicogenomic analysis revealed misexpression of Wnt signaling pathway genes in TCDD-exposed fin regenerates [43]. R-Spondin1, a potent activator of Wnt/ $\beta$-catenin signaling was highly expressed in fin tissue of TCDD-exposed fish following amputation. Although R-Spondin1 does not directly activate Wnt signaling, it interferes with Dkk/kremen-mediated internalization of the FZD coreceptor LRP5/6 resulting in increased LRP5/6 receptor density [34]. In support of this, we have prevented TCDD-induced inhibition of fin regeneration in loss of function experiments targeting R-Spondin1 or LRP5/6 [43]. This suggests that TCDD impairs regeneration through inappropriate activation of canonical Wnt signaling. These studies also highlight the utility of chemical genetics approaches in revealing novel molecular targets involved in tissue regeneration.

\section{FGF Signaling Is Involved in Wound Healing, Blastema Formation and Regenerative Outgrowth}

FGFs are members of a large family of short secreted polypeptides that bind heparin and induce dimerization/ activation of tyrosine kinase fgfr. FGF signaling is reportedly involved in mammalian wound healing, angiogenesis, embryonic development and heart, tail, limb and skeletal muscle regeneration $[1,12,44,45]$. There are several lines of evidence supporting a role for FGF signaling in epimorphic regeneration. In the adult fin model, fgfrl is expressed in mesenchymal cells located proximal to the wound epidermis [45]. Chemical impairment of fgfrl signaling reportedly blocks blastema formation and expression of the msx homeobox domain genes, msxb and msxc [45], which are putative markers of undifferentiated, proliferating cells in regenerating fin tissue [46]. In support of these findings, morpholino knockdown of fgfrl reduces msxb and msxc expression in the adult fin [47]. Additionally, knockdown of FGF signaling using a dominant negative fgfrl transgenic line impaired both heart and caudal fin regeneration $[16,17]$. Furthermore, a genetic screen for temperature-sensitive regeneration mutants revealed a devoid of blastema (dob) mutant that failed to 
initiate regeneration [48]. The dob mutant contains a null mutation in the ligand Fgf20a that results in the absence of mesenchymal proliferation during blastema formation. Fgf20a mutants display abnormalities in the wound epithelium following amputation, suggesting that FGF signaling regulates aspects of wound healing [48].

In addition to wound healing and blastema formation, FGF-dependent signaling has been implicated in patterning during regenerative outgrowth. Treatment with an fgfrl inhibitor following blastema formation $(48 \mathrm{~h}$ post amputation) inhibited regenerative outgrowth and decreased msxb and msxc expression in the regenerate [45]. Taken together, these studies provide strong evidence supporting a role for FGF signaling in wound healing, the initiation of blastema formation and regenerative outgrowth.

\section{Activin- $\beta A$ and RA Signaling Is Involved in Patterning}

The ability of cells to determine their position in three dimensions is critical for the establishment of proper patterning in a regenerating fin [49]. Some of the earliest work using the zebrafish caudal fin model has demonstrated an essential role for RA signaling in patterning during epimorphic regeneration. Retinoic acid receptor gamma (RAR gamma) is expressed in the blastema and exposure to RA during fin regeneration causes morphological changes in the regenerate $[50,51]$. RA inhibits regeneration by impacting the size of the wound epidermis, thereby impinging on patterning within the blastema [52], in addition to inducing apoptosis in the apical epithelial cap [53] (see Addendum).

Our group has recently conducted a large-scale chemical library screen to identify modulators of larval tissue regeneration [39]. We found that 5 members of the glucocorticoid family similarly impaired fin regeneration [39]. These data were confirmed using the model glucocorticoid receptor (GR) agonist beclomethasone dipropionate (BDP). Transient knockdown of GR activity by morpholino injection blocked BDP-induced impairment of fin regeneration [39]. These data indicate that the inhibitory effects of BDP exposure on tissue regeneration are GR dependent. In order to identify the molecular targets of inappropriate GR activation, we examined BDP-induced gene expression changes in regenerating fin tissue. Cripto (TDGF-1), an inhibitor of Activin- $\beta$-A signaling, was one of the most highly induced genes among the differentially expressed transcripts (S. Sengupta, unpublished observations). Using SB431542, an Activin signaling inhibitor, we confirmed that Activin signaling was critical for larval tissue regeneration (S. Sengupta, unpublished observations). Collectively, these data support the relevance of using chemical genetics to unravel the signaling pathways that dictate regeneration.

\section{Hedgehog Signaling Controls Skeletal Regeneration and Patterning}

Mainly through marker analysis, a number of genes are hypothesized to play a role in skeletal regeneration. Sonic hedgehog (shh) [54], its membrane-bound receptor patched1 (ptc1) [54], and bone morphogenic protein $2 \mathrm{~b}$ (bmp2b) [54, 55], bmp4 [55], and bmp6 [56] have been localized in the distal stump of each amputated ray in adult zebrafish regenerates (regenerated tissue). Blastema cells in contact with the epidermal layer differentiate into scleroblasts that ultimately give rise to connective and skeletal components of the regenerating fin tissue. Scleroblasts synthesize and release dermal bone matrix into the sub-epidermal space that is progressively mineralized to yield new skeletal structures [56]. Both shh and bmp signaling have been implicated in the regeneration of dermal bone [55, 56]. Quint et al. [55] showed that ectopic expression of shh or bmp2 in the blastema of regenerating fins resulted in excess bone deposition and abnormal patterning in the regenerate [55]. The same study reported that co-injection of exogenous shh with the bmp inhibitor chordin markedly reduced abnormal bone fusions induced by ectopic shh expression. A subsequent study from the same group demonstrated that injection of chordin failed to prevent shh and ptcl expression [56]. Together, these data imply that shh activation occurs upstream of BMP signaling with regard to patterning during regenerative outgrowth.

\section{Negative Regulators of Fin Regeneration}

Previous sections have highlighted the growing field devoted to unraveling the signaling pathways that initiate and promote fin regeneration. Beyond initiation, the regenerative process must also be tightly regulated to prevent mispatterning or appendage overgrowth. However, little is known regarding mechanisms that regulate signaling molecules that promote regeneration of those pathways that control termination of fin growth following amputation. It is thought that termination occurs by 
either an unknown active termination mechanism or by gradual cessation of regenerative signaling [22]. Fin regeneration proceeds rapidly until the preamputation fin length is reached, at which point it switches to an ontogenetic growth mechanism. Although the mechanisms that negatively regulate regeneration are unknown, a relatively small number of signaling molecules have been identified that play a role in the process.

In addition to wound healing, blastema formation and regenerative outgrowth, FGF-dependent signaling has been implicated in positional memory and patterning during regenerative outgrowth, thereby influencing regenerative termination. Positional memory is the term used to describe the rate of tissue regeneration coupled with the precise regulation of tissue regeneration such that only the appropriate structures are replaced [17]. Following amputation, Lee et al. [17] showed that different fin growth rates ensue dependent upon the location of the amputation plane relative to the distal tip of the fin. In the same study, use of a conditional dominant negative fgfrl zebrafish line driven by a heatshock promoter revealed that the level of FGF signaling determines the growth rate.

Noncanonical Wnt signaling has recently been implicated in negatively regulating regeneration following fin amputation. Specifically, noncanonical $\beta$-catenin-independent Wnt signaling was shown to block fin regeneration likely via impairment of $\mathrm{Wnt} / \beta$-catenin signaling [37]. Stoick-Cooper et al. [37] reported inhibition of regeneration, cell proliferation, and Wnt target gene expression in heat shock-inducible transgenic fish that overexpressed Wnt5b. Importantly, the same study showed that homozygous mutants for Wnt5b displayed increased rates of regeneration and increased fin size following regeneration relative to wild-type controls. The authors postulate that $\mathrm{Wnt} 5 \mathrm{~b}$ negatively regulates $\mathrm{Wnt} / \beta$-catenin signaling to regulate the size of the regenerate such that only the necessary structures and tissues are replaced.

Although the possibility of cross talk between FGF and Wnt signaling has been raised by others [37], a recent paper by Lee et al. [25] highlights both the restrictive and permissive roles of epidermal FGF signaling over Wnt signaling and blastemal proliferation in regenerating fin tissue. FGF signaling was shown to promote shh and lef1 expression in proximal epidermal cells, whereas wnt $5 b$ and possibly other inhibitory factors induced by FGF and Ras signaling function to restrict shh and lef1 expression from distal areas of the epidermis. This demonstrates the role of FGF and Wnt pathways in compartmentalizing hedgehog signaling, and, in turn, spatially regulating blastemal proliferation during regeneration.
In addition to noncanonical Wnt and FGF signaling, a recent paper has described the role of simplet (smp/ fam53b) in the regulation of skeletal regeneration in the fin. Morpholino knockdown of smp expression resulted in increased shh expression and inappropriate bone deposition in regenerating fin tissue [21]. Because shh is known to regulate bone patterning during regenerative outgrowth, the authors speculate that smp negatively regulates shh-dependent signaling [21]. Interestingly, in contrast to the study by Stoick-Cooper et al. [37], inhibition of smp signaling impaired regenerative outgrowth, suggesting that the mechanism by which smp influences positional memory is likely complex and may involve spatiotemporal considerations.

\section{Noncoding RNAs Negatively Regulate Epimorphic Regeneration}

MicroRNAs (miRNAs) are a class of noncoding RNAs that are $\sim 22$ nucleotide, single-stranded molecules characterized by a hairpin structure [57]. They have been identified as important endogenous posttranscriptional regulators controlling expression of approximately $60 \%$ of mammalian genes [58]. Following miRNA transcription and processing, one strand of the mature miRNA is incorporated into the RNA-induced silencing complex (RISC) [59]. The RISC-miRNA complex is guided to the 3' UTR of its target mRNA. Binding of miRNA to its complementary sites on target mRNA is followed by either de-adenylation and/or translational repression of target mRNAs leading to gene silencing [59]. Recently, two studies have shown that small noncoding RNAs regulate fin regeneration collectively identifying an immense new network of signaling players whose role in regeneration is not well understood [60, 61]. By knocking down dicer, Thatcher et al. [60] showed that miRNA biogenesis is necessary for regeneration in the adult fin model. The same study reported that miR-203 negatively regulates protein levels of the downstream Wnt signaling molecule Lef1, supporting the general hypothesis that downregulation of miRNAs permits the expression of target genes necessary for regeneration [60]. In a separate study, FGF-dependent depletion of miR-133 allowed expression of the miR-133 target gene mps1 kinase, a positive regulator of fin regeneration [62]. Taken together, these data support miRNA depletion as a mechanism for tissue generation and reveal a new level of regenerative signaling complexity. 


\section{Larval Fin Regeneration Model}

The adult fin regeneration model has unique advantages, but its usefulness as a regenerative model is limited by technical barriers such as the length of time necessary to raise adult fish (2-4 months) and allow for complete fin regeneration (12-14 days) as well as difficulty in achieving genetic manipulation in the fin. It is particularly challenging to study early signaling events that promote blastema formation because wound healing must occur prior to microinjection of antisense oligonucleotides that are commonly used in loss of function experiments. Recently, it was reported that the fin primordia is capable of complete regeneration (2-5 days after fertilization) similar to the adult zebrafish [63]. Morphologically, even in the absence of complete cellular differentiation, the larval fin regenerates similarly to the adult by the development of an apical wound epithelium followed by blastema formation, which later proliferates and differentiates into the required cell types [63]. Also, similar to the adult zebrafish, chemical inhibition of fgfrl by SU5402, aryl hydrocarbon receptor activation by TCDD and GR activation by beclomethasone abrogated larval fin regeneration $[39,41,42,45,63]$, suggesting that there are similarities at the cellular and molecular level between adult and larval regeneration. Mainly through marker analysis, Yoshinari et al. [64] showed that a number of genes including dlx5a, msxe, junbl, ilf2, mvp, phlda2, mmp9, hspa9, junb and smarca4 are expressed in conserved domains in both the larval and adult models (see Addendum). In summary, these data suggest that there is a high probability of common hierarchical molecular signaling pathways across different regeneration systems. While there are many powerful zebrafish regeneration models available, we believe that the larval caudal fin model is an outstanding regenerative platform because of the sheer number of embryos available daily in any zebrafish facility, its genetic tractability (access to the one-cell stage) and the ability to perform noninvasive amputations.

\section{Emerging Themes and Perspectives}

The field of regenerative medicine is aimed at awaking our latent ability to replace damaged tissues and structures by reactivating evolutionarily conserved signaling pathways that promote regeneration. Regenerative medicine is generally approached from two distinct angles. In recent years, stem cell-based models have been developed to generate a suite of differentiated cells suitable for im- plantation into patients. Alternatively, studies employing nonmammalian models designed to define the molecular events that permit tissue regeneration continue to identify signaling molecules that promote or suppress regenerative signaling for potential use in the clinic. Among the molecular networks discussed in this mini-review, FGF and Wnt signaling have emerged as two main signaling pathways that regulate virtually all aspects of epimorphic fin regeneration. Importantly, these signaling networks have been shown to be influential regulators of heart $[16$, 37], retina [65-67] and skeletal muscle $[68,69]$ regeneration and in the case of Wnt, liver [70, 71] and sensory hair cell [72] regeneration. Therefore, while the overall form and function of regenerating tissues may vary, some of the molecular mechanisms that dictate regeneration are likely conserved. Continuing studies designed to investigate how spatially restricted gene expression positively or negatively regulates regeneration, as well as how these molecular signaling networks interact with one another will be crucial to move the field forward. Ultimately, a better understanding of the complexity of signaling events that choreograph epimorphic regeneration will provide new avenues for comparative studies in mammalian models with the hope of developing novel therapeutics to slow or prevent tissue loss from aging, injury and disease.

\section{Acknowledgements}

We thank Katrine Saili, Britton Goodale, Sumitra Sengupta and Michael Simonich for helpful discussions and the critical review of this manuscript. T.L.T. and J.A.F. are recipients of the Environmental and Molecular Toxicology Training Grant T32 ES07060. This research was supported by the NIEHS Environmental Health Sciences Center Grant No. ES00210 and NSF Grant No. 0641409 .

References

1 Poss KD, Wilson LG, Keating MT: Heart regeneration in zebrafish. Science 2002;298: 2188-2190.

$\checkmark 2$ Raya A, Consiglio A, Kawakami Y, Rodriguez-Esteban C, Izpisua-Belmonte JC: The zebrafish as a model of heart regeneration. Cloning Stem Cells 2004;6:345-351.

-3 Johnson SL, Weston JA: Temperature-sensitive mutations that cause stage-specific defects in zebrafish fin regeneration. Genetics 1995;141:1583-1595

4 Bernhardt RR, Tongiorgi E, Anzini P, Schachner M: Increased expression of specific recognition molecules by retinal ganglion cells and by optic pathway glia accompanies the successful regeneration of retinal axons in adult zebrafish. J Comp Neurol 1996;376:253-264. 
5 Becker CG, Becker T: Repellent guidance of regenerating optic axons by chondroitin sulfate glycosaminoglycans in zebrafish. J Neurosci 2002;22:842-853.

-6 Sadler KC, Krahn KN, Gaur NA, Ukomadu C: Liver growth in the embryo and during liver regeneration in zebrafish requires the cell cycle regulator, uhrf1. Proc Natl Acad Sci USA 2007;104:1570-1575.

$\checkmark 7$ Becker CG, Lieberoth BC, Morellini F, Feldner J, Becker T, Schachner M: L1.1 is involved in spinal cord regeneration in adult zebrafish. J Neurosci 2004;24:7837-7842.

$\checkmark 8$ Lopez-Schier H, Hudspeth AJ: A two-step mechanism underlies the planar polarization of regenerating sensory hair cells. Proc Natl Acad Sci USA 2006;103:18615-18620.

$\checkmark 9$ Pueyo JI, Couso JP: Parallels between the proximal-distal development of vertebrate and arthropod appendages: homology without an ancestor? Curr Opin Genet Dev 2005; 15:439-446.

10 Geetha-Loganathan P, Nimmagadda S, Scaal $\mathrm{M}$ : Wnt signaling in limb organogenesis. Organogenesis 2008;4:109-115.

11 Mariani FV, Ahn CP, Martin GR: Genetic evidence that FGFs have an instructive role in limb proximal-distal patterning. Nature 2008;453:401-405.

-12 Seed J, Olwin BB, Hauschka SD: Fibroblast growth factor levels in the whole embryo and limb bud during chick development. Dev Biol 1988;128:50-57.

13 Poss KD, Keating MT, Nechiporuk A: Tales of regeneration in zebrafish. Dev Dyn 2003; 226:202-210.

-14 Makino S, Whitehead GG, Lien CL, Kim S, Jhawar P, Kono A, Kawata Y, Keating MT: Heat-shock protein 60 is required for blastema formation and maintenance during regeneration. Proc Natl Acad Sci USA 2005; 102:14599-14604.

-15 Qin Z, Barthel LK, Raymond PA: Genetic evidence for shared mechanisms of epimorphic regeneration in zebrafish. Proc Natl Acad Sci USA 2009;106:9310-9315.

-16 Lepilina A, Coon AN, Kikuchi K, Holdway JE, Roberts RW, Burns CG, Poss KD: A dynamic epicardial injury response supports progenitor cell activity during zebrafish heart regeneration. Cell 2006;127:607-619.

17 Lee Y, Grill S, Sanchez A, Murphy-Ryan M, Poss KD: FGF signaling instructs positiondependent growth rate during zebrafish fin regeneration. Development 2005;132:51735183.

18 Lien CL, Schebesta M, Makino S, Weber GJ, Keating MT: Gene expression analysis of zebrafish heart regeneration. PLoS Biol 2006;4: 260.

-19 Schebesta M, Lien CL, Engel FB, Keating MT: Transcriptional profiling of caudal fin regeneration in zebrafish. ScientificWorldJournal 2006;6(suppl 1):38-54.

-20 Raya A, Koth CM, Buscher D, Kawakami Y, Itoh T, Raya RM, Sternik G, Tsai HJ, Rodriguez-Esteban C, Izpisua-Belmonte JC: Acti- vation of Notch signaling pathway precedes heart regeneration in zebrafish. Proc Natl Acad Sci USA 2003; 100(suppl 1):1188911895.

-21 Kizil C, Otto GW, Geisler R, Nusslein-Volhard C, Antos CL: Simplet controls cell proliferation and gene transcription during zebrafish caudal fin regeneration. Dev Biol 2009;325:329-340.

22 Iovine MK: Conserved mechanisms regulate outgrowth in zebrafish fins. Nat Chem Biol 2007;3:613-618.

-23 Akimenko MA, Mari-Beffa M, Becerra J, Geraudie J: Old questions, new tools, and some answers to the mystery of fin regeneration. Dev Dyn 2003;226:190-201.

-24 Nechiporuk A, Keating MT: A proliferation gradient between proximal and msxb-expressing distal blastema directs zebrafish fin regeneration. Development 2002;129:26072617.

25 Lee Y, Hami D, De Val S, Kagermeier-Schenk B, Wills AA, Black BL, Weidinger G, Poss KD: Maintenance of blastemal proliferation by functionally diverse epidermis in regenerating zebrafish fins. Dev Biol 2009;331: 270-280.

26 Huelsken J, Behrens J: The Wnt signalling pathway. J Cell Sci 2002;115:3977-3978.

27 Kikuchi A, Yamamoto H, Kishida S: Multiplicity of the interactions of Wnt proteins and their receptors. Cell Signal 2007;19:659671.

28 Kikuchi A, Yamamoto H, Sato A: Selective activation mechanisms of Wnt signaling pathways. Trends Cell Biol 2009;19:119-129.

29 Liu G, Bafico A, Aaronson SA: The mechanism of endogenous receptor activation functionally distinguishes prototype canonical and noncanonical Wnts. Mol Cell Biol 2005;25:3475-3482.

- 30 Hsieh JC, Kodjabachian L, Rebbert ML, Rattner A, Smallwood PM, Samos CH, Nusse R, Dawid IB, Nathans J: A new secreted protein that binds to Wnt proteins and inhibits their activities. Nature 1999;398:431-436.

- 31 Glinka A, Wu W, Delius H, Monaghan AP, Blumenstock C, Niehrs C: Dickkopf-1 is a member of a new family of secreted proteins and functions in head induction. Nature 1998;391:357-362.

32 Semenov MV, Tamai K, Brott BK, Kuhl M, Sokol S, He X: Head inducer dickkopf-1 is a ligand for Wnt coreceptor lrp6. Curr Biol 2001;11:951-961.

33 Mao B, Wu W, Li Y, Hoppe D, Stannek P, Glinka A, Niehrs C: Ldl-receptor-related protein 6 is a receptor for dickkopf proteins. Nature 2001;411:321-325.

34 Binnerts ME, Kim KA, Bright JM, Patel SM, Tran K, Zhou M, Leung JM, Liu Y, Lomas WE, 3rd, Dixon M, Hazell SA, Wagle M, Nie WS, Tomasevic N, Williams J, Zhan X, Levy MD, Funk WD, Abo A: R-Spondin1 regulates Wnt signaling by inhibiting internalization of LRP6. Proc Natl Acad Sci USA 2007;104:14700-14705.
35 Poss KD, Shen J, Keating MT: Induction of lef1 during zebrafish fin regeneration. Dev Dyn 2000;219:282-286.

-36 Kawakami Y, Rodriguez Esteban C, Raya M, Kawakami H, Marti M, Dubova I, Izpisua Belmonte JC: Wnt/beta-catenin signaling regulates vertebrate limb regeneration. Genes Dev 2006;20:3232-3237.

- 37 Stoick-Cooper CL, Weidinger G, Riehle KJ, Hubbert C, Major MB, Fausto N, Moon RT: Distinct Wnt signaling pathways have opposing roles in appendage regeneration. Development 2007;134:479-489.

38 Chen B, Dodge ME, Tang W, Lu J, Ma Z, Fan CW, Wei S, Hao W, Kilgore J, Williams NS, Roth MG, Amatruda JF, Chen C, Lum L: Small molecule-mediated disruption of Wnt-dependent signaling in tissue regeneration and cancer. Nat Chem Biol 2009;5: 100-107.

- 39 Mathew LK, Sengupta S, Kawakami A, Andreasen EA, Lohr CV, Loynes CA, Renshaw SA, Peterson RT, Tanguay RL: Unraveling tissue regeneration pathways using chemical genetics. J Biol Chem 2007;282:3520235210.

40 Mathew LK, Simonich MT, Tanguay RL: AHR-dependent misregulation of Wnt signaling disrupts tissue regeneration. Biochem Pharmacol 2009;77:498-507.

41 Mathew LK, Andreasen EA, Tanguay RL: Aryl hydrocarbon receptor activation inhibits regenerative growth. Mol Pharmacol 2006;69:257-265.

42 Zodrow JM, Tanguay RL: 2,3,7,8-tetrachlorodibenzo-p-dioxin inhibits zebrafish caudal fin regeneration. Toxicol Sci 2003;76: 151-161.

43 Mathew LK, Sengupta SS, Ladu J, Andreasen EA, Tanguay RL: Crosstalk between AHR and Wnt signaling through R-Spondin1 impairs tissue regeneration in zebrafish. FASEB J 2008;22:3087-3096.

44 Gospodarowicz D: Humoral control of cell proliferation: the role of fibroblast growth factor in regeneration, angiogenesis, wound healing, and neoplastic growth. Prog Clin Biol Res 1976;9:1-19.

45 Poss KD, Shen J, Nechiporuk A, McMahon G, Thisse B, Thisse C, Keating MT: Roles for Fgf signaling during zebrafish fin regeneration. Dev Biol 2000;222:347-358.

46 Akimenko MA, Johnson SL, Westerfield M, Ekker M: Differential induction of four msx homeobox genes during fin development and regeneration in zebrafish. Development 1995;121:347-357.

47 Thummel R, Bai S, Sarras MP Jr, Song P, McDermott J, Brewer J, Perry M, Zhang X, Hyde DR, Godwin AR: Inhibition of zebrafish fin regeneration using in vivo electroporation of morpholinos against fgfr1 and msxb. Dev Dyn 2006;235:336-346.

-48 Whitehead GG, Makino S, Lien CL, Keating MT: fgf20 is essential for initiating zebrafish fin regeneration. Science 2005;310:19571960. 
-49 Stoick-Cooper CL, Moon RT, Weidinger G: Advances in signaling in vertebrate regeneration as a prelude to regenerative medicine. Genes Dev 2007;21:1292-1315.

50 White JA, Boffa MB, Jones B, Petkovich M: A zebrafish retinoic acid receptor expressed in the regenerating caudal fin. Development 1994;120:1861-1872.

51 Geraudie J, Monnot MJ, Brulfert A, Ferretti P: Caudal fin regeneration in wild type and long-fin mutant zebrafish is affected by retinoic acid. Int J Dev Biol 1995;39:373-381.

52 Ferretti P, Geraudie J: Retinoic acid-induced cell death in the wound epidermis of regenerating zebrafish fins. Dev Dyn 1995;202: 271-283.

53 Geraudie J, Ferretti P: Correlation between RA-induced apoptosis and patterning defects in regenerating fins and limbs. Int J Dev Biol 1997;41:529-532.

54 Laforest L, Brown CW, Poleo G, Geraudie J, Tada M, Ekker M, Akimenko MA: Involvement of the sonic hedgehog, patched 1 and bmp2 genes in patterning of the zebrafish dermal fin rays. Development 1998;125: 4175-4184.

55 Quint E, Smith A, Avaron F, Laforest L, Miles J, Gaffield W, Akimenko MA: Bone patterning is altered in the regenerating zebrafish caudal fin after ectopic expression of sonic hedgehog and bmp2b or exposure to cyclopamine. Proc Natl Acad Sci USA 2002; 99:8713-8718.

56 Smith A, Avaron F, Guay D, Padhi BK, Akimenko MA: Inhibition of bmp signaling during zebrafish fin regeneration disrupts fin growth and scleroblasts differentiation and function. Dev Biol 2006;299:438-454.

57 Wienholds E, van Eeden F, Kosters M, Mudde J, Plasterk RH, Cuppen E: Efficient target-selected mutagenesis in zebrafish. Genome Res 2003;13:2700-2707.

58 Friedman RC, Farh KK, Burge CB, Bartel DP: Most mammalian mRNAs are conserved targets of microRNAs. Genome Res 2009; 19:92-105.

59 Williams AE: Functional aspects of animal microRNAs. Cell Mol Life Sci 2008;65:545562 .
60 Thatcher EJ, Paydar I, Anderson KK, Patton JG: Regulation of zebrafish fin regeneration by microRNAs. Proc Natl Acad Sci USA 2008;105:18384-18389.

61 Yin VP, Thomson JM, Thummel R, Hyde DR, Hammond SM, Poss KD: Fgf-dependent depletion of microRNA-133 promotes appendage regeneration in zebrafish. Genes Dev 2008;22:728-733.

62 Yin VP, Poss KD: New regulators of vertebrate appendage regeneration. Curr Opin Genet Dev 2008;18:381-386.

63 Kawakami A, Fukazawa T, Takeda H: Early fin primordia of zebrafish larvae regenerate by a similar growth control mechanism with adult regeneration. Dev Dyn 2004;231:693699.

64 Yoshinari N, Ishida T, Kudo A, Kawakami A: Gene expression and functional analysis of zebrafish larval fin fold regeneration. Dev Biol 2009;325:71-81.

65 Del Rio-Tsonis K, Jung JC, Chiu IM, Tsonis PA: Conservation of fibroblast growth factor function in lens regeneration. Proc Natl Acad Sci USA 1997;94:13701-13706.

-66 Osakada F, Ooto S, Akagi T, Mandai M, Akaike A, Takahashi M: Wnt signaling promotes regeneration in the retina of adult mammals. J Neurosci 2007;27:4210-4219.

67 Petersen CP, Reddien PW: Smed-betacatenin-1 is required for anteroposterior blastema polarity in planarian regeneration. Science 2008;319:327-330.

68 Floss T, Arnold HH, Braun T: A role for FGF6 in skeletal muscle regeneration. Genes Dev 1997;11:2040-2051.

69 Otto A, Schmidt C, Luke G, Allen S, Valasek P, Muntoni F, Lawrence-Watt D, Patel K: Canonical Wnt signalling induces satellite-cell proliferation during adult skeletal muscle regeneration. J Cell Sci 2008;121:29392950.

70 Goessling W, North TE, Loewer S, Lord AM, Lee S, Stoick-Cooper CL, Weidinger G, Puder M, Daley GQ, Moon RT, Zon LI: Genetic interaction of PGE2 and Wnt signaling regulates developmental specification of stem cells and regeneration. Cell 2009;136:11361147.
71 Monga SP, Pediaditakis P, Mule K, Stolz DB, Michalopoulos GK: Changes in Wnt/betacatenin pathway during regulated growth in rat liver regeneration. Hepatology 2001;33: 1098-1109.

72 Hawkins RD, Bashiardes S, Powder KE, Sajan SA, Bhonagiri V, Alvarado DM, Speck J, Warchol ME, Lovett M: Large scale gene expression profiles of regenerating inner ear sensory epithelia. PLoS One 2007;2:525.

\section{Addendum}

We recently conducted a comparative analysis of genes similarly regulated during larval fin and adult fin and heart regeneration. Interestingly, raldh2, a ratelimiting enzyme necessary for RA synthesis, was one of the most highly expressed genes across all three regeneration pathways. Both chemical inhibition and antisense oligonucleotide morpholino knockdown of raldh2 prevented regeneration while application of exogenous RA markedly rescued inhibition of regeneration. Collectively, these data demonstrate a clear role for RA signaling in epimorphic regeneration. This study also showed that $64 \%$ of the genes that are misregulated during the early stages of regeneration ( $1 \mathrm{dpa}$ ) are conserved between nonamputated control and regenerating fin tissue in the adult and larval fin regeneration models.

\section{Reference}

1 Mathew LK, Sengupta S, Franzosa JA, Perry J, La Du J, Andreasen EA, Tanguay RL: Comparative expression profiling reveals an essential role for Raldh2 in epimorphic regeneration. J Biol Chem 2009; doi: 10.1074/jbc. M109.011668. 